Discussion Paper No. 05-19

\title{
Impure Public Goods and Technological Interdependencies
}

Andreas Löschel and Dirk T.G. Rübbelke

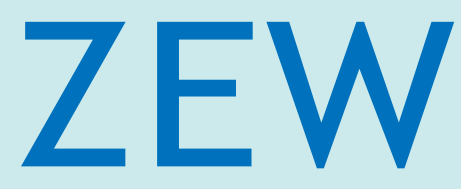

Zentrum für Europäische Wirtschaftsforschung $\mathrm{GmbH}$

Centre for European

Economic Research 
Discussion Paper No. 05-19

\title{
Impure Public Goods and Technological Interdependencies
}

\author{
Andreas Löschel and Dirk T.G. Rübbelke
}

Download this ZEW Discussion Paper from our ftp server:

ftp://ftp.zew.de/pub/zew-docs/dp/dp0519.pdf

Die Discussion Papers dienen einer möglichst schnellen Verbreitung von neueren Forschungsarbeiten des ZEW. Die Beiträge liegen in alleiniger Verantwortung der Autoren und stellen nicht notwendigerweise die Meinung des ZEW dar.

Discussion Papers are intended to make results of ZEW research promptly available to other economists in order to encourage discussion and suggestions for revisions. The authors are solely responsible for the contents which do not necessarily represent the opinion of the ZEW. 


\section{Nontechnical Summary}

In economics public goods are regularly treated as pure public goods, although most public goods are not "purely public". The reason for doing so most likely lies in the simplicity of pure public goods analysis. In contrast, the analysis of public goods which are not purely public tends to become rather complex.

Pioneering work with respect to the issue of impure public goods has been provided by Cornes and Sandler (1984), who developed an approach to analyze impure public goods where the considered consumer's utility function is defined over three characteristics. They suggested to apply this approach to an activity like philanthropy. This idea has later been elaborated and initiated a new strand of literature which is associated with the expression "warm-glow giving". Many further analyses of impure public goods followed which were based on the approach suggested by Cornes and Sandler. Results were obtained which could not be observed by employing the simple pure public good approach. So, Cornes and Sandler (1994) illustrated in a comparative static analysis that quite surprising results may arise within an impure public good model. In fact, in their modelling, they analyzed the joint production of characteristics of different degrees of publicness. Their analysis demonstrated that substitutability and complementarity of the private and pure public characteristics generated by the public good play a crucial role with respect to comparative static responses. Joint characteristic or joint production models have been used to analyze, e.g., climate protection, environmentally friendly consumption, financing of public radio stations, military alliances, refuse collection, and terrorism. Probably almost every global public good provision represents a joint production of several characteristics of different degrees of publicness, i.e., global public goods production is an impure public goods production.

Although impure public good models found application in many fields of economics, one issue has been widely neglected until recently: the effect of alternative technologies on the models' results. The neglect of alternative technologies may cause an overstatement of the benefits of a joint production technology and may therefore, result in an inefficient level of joint production. Let us consider the example of climate policy. A climate policy which raises energy efficiency, for example, tends to reduce the required level of fossil fuels to be combusted. This reduction is, on the one hand, associated with a mitigation of greenhouse gas emissions and, consequently, provides climate-protection and primary benefits. On the other hand, the reduction is associated with ancillary effects in the shape of, e.g., a decline in other pollutants' emissions like sulphur dioxide emissions. Several studies predict that the associated ancillary benefits even exceed the primary benefits. However, regularly, it is at least implicitly postulated that the ancillary benefits can only be acquired by climate policies. Yet, desulphurisation installations would also be an effective means to reduce sulphur dioxide emissions and these installations do not protect the climate. A priori, it is not clear from a welfare economics' point of view whether it is more efficient to mitigate sulphur dioxide by means of desulphurisation installations or by means of climate policies, since the implementation costs of the different technologies may diverge. Provided a country would consider desulphurisation installations to be more 
attractive, an augmented use of such installations would reduce the ancillary benefits of climate policy and, therefore, would tend to reduce total benefits of climate protection. This, again, mitigates the attractiveness of climate policy. As this example discloses, quantities and prices/costs of the alternative technologies exclusively producing the private characteristic, are crucial for the ancillary benefits of climate policy to be expected, and consequently, for the efficient policy design. Changes in these parameters have an impact on the efficient impure public good level, in our example, on the optimal climate policy level.

In our paper we present a more general setting of the comparative statics model suggested by Cornes and Sandler (1994). The integration of an alternative technology into the comparative statics model, however, requires the analysis of three instead of two commodities. By this extension different levels of substitutability between the jointly provided private goods/characteristics and other private goods can be captured. Only a certain group of private goods is considered to represent an adequate substitute for the joint production's private characteristic, and this group is assumed to be regulated by a central authority. As we show, these extensions are sensible for analyzing several real-world impure-public-goods examples. The focus of the comparative statics analysis is on the two parameters directly associated with the substitute: its prices and quantities. In order to check and refine the results of the analytical study, we employed a simulation model for the example of climate policy in Germany. Here, climate protection represents the global public characteristic of the impure public good "climate policy", while sulphur dioxide abatement represents the private characteristic, from an individual country's point of view. In fact, most options to reduce the emission levels of the greenhouse gas $\mathrm{CO}_{2}$ turn out to be simultaneously reducing the emissions of sulphur dioxide. However, the emissions of sulphur dioxide can also be mitigated independently of climate policy by means of desulphurisation installations, which represent the technology independently generating the private characteristic of the impure public good. The results of the simulations confirmed the findings of the analytical model. So, the increase of the price of climate-policy independent sulphur dioxide abatement exerts a negative income effect. Consequently, the abatement has a negative impact on impure public good provision, and therefore on climate protection levels. As our explicit illustration of welfare effects in the framework of the simulation model shows, welfare levels unambiguously decline with the considered price increases. In contrast to the ambiguous results of our analytical representation with respect to the impacts of an increase in the provision of the rationed good, we found unambiguous effects of such an increase in our simulation model. The provision of the impure public good decrease with increasing levels of the rationed private good. Due to the inefficiently low provision level in the baseline scenario, the increased production of the rationed good caused an improvement of welfare. Interestingly, the impact of the price increase of sulphur dioxide abatement has minor effects on climate protection levels compared to increases in the climate-policy-independent enhancement of sulphur dioxide abatement. Consequently, it turns out to be likely that stricter regulations on air-pollution may cause the attractiveness of climate policy to decline significantly, while price increases of this control tend to have only minor effects. 


\title{
Impure Public Goods and Technological Interdependencies
}

\author{
Andreas Löschel* and Dirk T.G. Rübbelke ${ }^{\dagger}$
}

March 2005

\begin{abstract}
Impure public goods represent an important group of goods. Almost every public good exerts not only effects which are public to all but also effects which are private to the producer of this good. What is often omitted in the analysis of impure public goods is the fact that - regularly - these private effects can also be generated independently of the public good.

In our analysis we focus on the effects alternative technologies - independently generating the private effects of the public good - may have on the provision of impure public goods. After the investigation in an analytical impure public good model, we numerically simulate the effects of alternative technologies in a parameterized model for climate policy in Germany.
\end{abstract}

Keywords: impure public goods, climate policy, rationing

JEL classification: H41, C61, Q53, Q54

*Zentrum für Europäische Wirtschaftsforschung GmbH L 7,1, 68161 Mannheim, Germany, email: loeschel@zew.de

${ }^{\dagger}$ Department of Economics, Chemnitz University of Technology, 09107 Chemnitz, Germany, email: dirk.ruebbelke@wirtschaft.tu-chemnitz.de 


\section{Introduction}

In economics public goods are regularly treated as pure public goods, although most public goods are not "purely public". The reason for doing so most likely lies in the simplicity of pure public goods analysis. In contrast, the analysis of public goods which are not purely public tends to become rather complex.

Pioneering work with respect to the issue of impure public goods has been provided by Cornes and Sandler (1984), who developed an approach to analyze impure public goods where the considered consumer's utility function is defined over three characteristics. They suggested to apply this approach to an activity like philanthropy. This idea has later been elaborated by Andreoni (1986, 1989, 1990). Andreoni's works initiated a new strand of literature which is associated with the expression "warm-glow giving".

Many further analyses of impure public goods followed which were based on the approach suggested by Cornes and Sandler. Results were obtained which could not be observed by employing the simple pure public good approach. So, Cornes and Sandler (1994) illustrated in a comparative static analysis that quite surprising results may arise within an impure public good model. In fact, in their modelling, they analyzed the joint production of characteristics of different degrees of publicness. Their analysis demonstrated that substitutability and complementarity of the private and pure public characteristics generated by the public good play a crucial role with respect to comparative static responses. Such effects could - of course - not be observed in pure public good models, since these models exclusively focus on the public good's pure public characteristic and disregard private ancillary characteristics of public goods. Ihori $(1992,1994)$ analyses some further comparative static properties of impure public good models, but his modelling differs from the one suggested by Cornes and Sandler (1994).

And despite its complexity, joint production and impure public goods modelling became an attractive device in the economics discipline. Joint characteristic or joint production models have been used to analyze, e.g., climate protection (Sandler (1996), Rübbelke (2003)), environmentally friendly consumption (Kotchen (2005)), financing of public radio stations (Kingma and McClelland (1995)), military alliances (Sandler and Murdoch (1990), Sandler and Hartley (2001)), refuse collection (Dubin and Navarro (1988)), and terrorism (Rübbelke (2005)).

Additional interest in impure public goods models may arise because of the topicality of the global public goods discussion (Kaul et al. (1999)). Probably almost every global 
public good provision represents a joint production of several characteristics of different degrees of publicness, i.e., global public goods production is an impure public goods production. ${ }^{1}$

So, preservation of tropical forests may be considered as a global impure public good. A pure public characteristic of this public good is the preservation of biodiversity which provides genetic material that may contain cures for diseases representing dangers for all humans. Another pure public characteristic is the global climate protecting impact of the forests' carbon storage. But as Sandler (1997: 97) points out: "Not all of the benefits of the forests are global public goods; many are either private goods or public goods whose benefits are either specific to the host nation or to neighboring nations." Such private benefits are derived, e.g., from reduced soil erosion and preservation of watersheds.

Reducing poverty by raising foreign aid also provides several characteristics of different degrees of publicness. In March 2002, US President George W. Bush announced the first significant raise in US development assistance in a decade. This represents a 50 percent increase over current development assistance (Diamond 2002: 2). Before, the US aid budget has fallen from 0.24 percent of GDP in the mid-1980s to 0.1 percent in 2002 . The recent sharp increase in the US foreign aid has been justified by the claim that the reduction of poverty will combat terrorism. ${ }^{2}$ Therefore, we face a global public characteristic which we may denote "international security". Furthermore, the reduction of poverty by paying transfers generates monetary benefits in the poor regions. These benefits are mainly perceived regionally and are 'private' to the transfer-receiving region (the transfers can be employed to improve national infrastructure, to construct new hospitals or schools etc.).

The same line of reasoning can be applied to other global issues like climate protection, international combat of diseases or protection of international waters. Examples of impure public goods of smaller geographical dimension are, e.g., theaters or universities. Of course, there is an audience enjoying shows in the theater. However, according to Baumol and Bowen (1966), performing arts may also raise local identity and international prestige. Hence, we observe a kind of private (enjoyed exclusively by the audience) as well as a locally/regionally public (enjoyed by the whole region) characteristic. Similarly, students

\footnotetext{
${ }^{1}$ Sandler and Hartley (2001: 880) even stress: "The joint product model is relevant for virtually every public good scenario." And joint production is in general associated with characteristics of different degrees of publicness.

${ }^{2}$ President Bush pointed out in his speech at the United Nations Financing for Development Conference in Monterrey, Mexico: "We fight against poverty because hope is an answer to terror."
} 
get education in universities (private characteristic), but a university also provides benefits to its whole region by attracting companies (regionally public characteristic).

Although impure public good models found application in many fields of economics, one issue has been widely neglected until recently: the effect of alternative technologies on the models' results. The neglect of alternative technologies may cause an overstatement of the benefits of a joint production technology and may therefore result in an inefficient level of joint production. Let us consider the example of climate policy. A climate policy which raises energy efficiency, for example, tends to reduce the required level of fossil fuels to be combusted. This reduction is, on the one hand, associated with a mitigation of greenhouse gas emissions and, consequently, provides climate-protection and primary benefits. ${ }^{3}$ On the other hand, the reduction is associated with ancillary effects in the shape of, e.g., a decline in other pollutants' emissions like $\mathrm{SO}_{2}$ emissions. Several studies predict that the associated ancillary benefits even exceed the primary benefits. ${ }^{4}$ However, regularly, it is at least implicitly postulated that the ancillary benefits can only be acquired by climate policies. Yet, desulphurisation installations would also be an effective means to reduce $\mathrm{SO}_{2}$ emissions and these installations do not protect the climate. A priori, it is not clear from a welfare economics' point of view whether it is more efficient to mitigate $\mathrm{SO}_{2}$ by means of desulphurisation installations or by means of climate policies, since the implementation costs of the different technologies may diverge. ${ }^{5}$ Provided a country would consider desulphurisation installations to be more attractive, an augmented use of such installations would reduce the ancillary benefits of climate policy and, therefore, would tend to reduce total benefits of climate protection. This, again, mitigates the attractiveness of climate policy. ${ }^{6}$ As this example discloses, quantities and prices/costs of the alternative technologies exclusively producing the private characteristic, are crucial for the ancillary benefits of climate policy to be expected, and consequently, for the efficient policy design. Changes in these parameters have an impact on the efficient impure public good level, in our example, on the optimal climate policy level.

One of the rare exceptions in the literature that takes account of the role of alternative technologies, is the joint supply model suggested by Posnett and Sandler (1986).

\footnotetext{
${ }^{3}$ Since climate protection is the primary aim of climate policy, the benefits derived from climate protection are called the primary benefits of climate policy.

${ }^{4}$ For an overview of studies and ratios between primary and ancillary benefits, see Pearce (2000).

${ }^{5}$ Of course, if equal cost would prevail, the climate policy option is more attractive, since it additionally provides primary benefits.

${ }^{6}$ Yet, in the case where joint products are strong complements, different and quite surprising demand responses can be observed, see Cornes and Sandler (1994).
} 
They analyze a consumer's decision between two different commodities. One commodity is a private good whose purchase is associated with and tied to the finance of a public output (joint production). The other commodity is a purely private good. Therefore, they consider two different technologies providing private good consumption, one which is associated with joint production and one without joint production. This idea is now revived in Section 2, where we present a more general setting of the comparative statics model suggested by Cornes and Sandler (1994). ${ }^{7}$ The integration into the comparative statics model, however, requires the analysis of three instead of two commodities. By this extension different levels of substitutability between the jointly provided private goods/characteristics and other private goods can be captured, i.e. heterogeneity among private goods/characteristics is taken into account. Only a certain group of private goods is considered to represent an adequate substitute for the joint production's private characteristic, and this group is assumed to be regulated by a central authority. As we will show, these extensions are sensible for analyzing several real-world impure-public-goods examples. The focus of the comparative statics analysis in Section 3 will be on the two parameters directly associated with the substitute: its prices and quantities.

Afterwards, in Section 4, our paper goes beyond the analytical analysis of comparative statics by replicating the analytical approach into the sphere of numerical simulation. We employ the impure-public-good example of climate policy in Germany in order to simulate the comparative statics in the framework of a parameterized simulation model.

Section 5 stresses the main findings of the simulation model and compares them to the analytical findings. Results which could be found in the simulations but not in the analytical model are highlighted. Section 6 concludes.

\section{An Impure Public Good Model}

In the subsequent sections, an agent's decision on public good provision is analyzed in an impure public good model, where the comparative static properties are investigated with respect to modifications in the impure-public-good independent production technology of the private characteristic which is also generated by the public good. The agent's responses are expressed in terms of parameters associated with standard price taking functions.

\footnotetext{
${ }^{7}$ This modification has first been suggested by Rübbelke $(2002,2003)$.
} 


\subsection{Characteristics of Impure Public Goods}

Similar to Posnett and Sandler (1986), we consider an impure public good whose private characteristic can also be generated independently of the public characteristic. We analyze the impact of the independent generation of the joint production's private characteristic on agents' willingness to provide the impure public good. Hence, the level of the independent generation as well as its price have to be considered. In order to analyze the impact of the independent generation of the private characteristic on agents' willingness to provide the public good, we have to formulate an agent's utility maximization problem in a way that takes explicit account of the characteristics of the joint production. Agents' preferences are not expressed in commodity space but in characteristic space, i.e. not the amount of commodities acquired but the amount of characteristics enjoyed are crucial for the agents' maximization problems. ${ }^{8}$ We distinguish the characteristics provided by the impure public good according to their degree of publicness:

Pure public characteristic: No agent of the considered 'model world' can be excluded from its consumption and it exhibits non-rivalry.

Private characteristic: The private characteristic of an impure public good provision can be exclusively enjoyed by the agent making this provision. The public good contributing agent may be an individual, a region or a country.

\subsection{Utility Function and Characteristics}

We consider an agent whose preferences with respect to the consumption of three characteristics - $y_{1}, y_{2}$ and $X$ - are represented by a continuous utility function, $U\left(y_{1}, y_{2}, X\right)$, that is strictly increasing and strictly quasi-concave and everywhere twice differentiable. The country consumes three commodities:

First private good: Each unit of the first generates one unit of $y_{1}$ for the country at a price $p_{y}=1$. The commodity represents a bundle of private goods to the country and it may be thought of to be identical to the characteristic $y_{1}$. The symbol $y_{1}$ can consequently be used to denote either the first characteristic or the good that generates it. $y_{1}$ is used as the numeraire good in all that follows.

Second private good: The second commodity $s$ generates the private characteristic $y_{2}$.

\footnotetext{
${ }^{8}$ For a distinction of characteristics and commodities see Cornes (1992: 139).
} 
The generated characteristic $y_{2}$ is identical to the one which arises from the impure public good. One unit of $s$ is assumed to have the price $p_{s}$ and to generate one unit of characteristic $y_{2}$. Therefore, $s$ does not only denote the consumption of the second commodity, it also stands for the amount of characteristic $y_{2}$ generated by this commodity.

Impure public good: The third good $q$ represents the impure public good and has a price $p_{q}$. Each unit generates $\beta$ units of the public characteristic $X$. Furthermore, the impure public good additionally produces $\alpha$ units of the private characteristic $y_{2}$.

The total consumption of the public characteristic $X$ by agent $i$ equals the sum of his own contribution $x_{i}=\beta q_{i}$ and the other $n-1$ agents' contributions $\tilde{X}_{i}=\beta \tilde{Q}_{i}$ :

$$
X=x_{1}+x_{2}+\ldots+x_{n}=\sum_{j=1}^{n} x_{j}=x_{i}+\tilde{X}_{i}=\beta \sum_{j=1}^{n} q_{j}=\beta\left(q_{i}+\tilde{Q}_{i}\right)
$$

\subsection{Constraints Limiting the Welfare Maximization}

There are three constraints limiting the feasible consumption set from which the agent chooses his most preferred point:

- The first constraint is represented by the conventional budget constraint:

$$
y_{1}+p_{s} s+p_{q} q=I
$$

with $p_{q}>p_{s}$. Hence, the agent spends his whole income on the consumption of the three commodities.

- The Nash-Cournot assumption is made that an individual agent takes the other agents' generation of the public characteristic

$$
\tilde{X}=X-\beta q
$$

as exogenously given. Furthermore, the parameter $\beta$ is considered to be the same across all agents, which is equivalent to assuming that $\tilde{Q}$ is taken as given.

- We assume that an agent has to provide an exogenously fixed quantity of $s$ units of characteristic $y_{2}$ directly. It is assumed that this obligation is laid down by a national or international regulation. The unit cost of $s$ is also assumed to be exogenously determined. Therefore,

$$
s=\bar{s} \quad \text { and } \quad p_{s}=\overline{p_{s}} .
$$


In the formulation of the agent's consumption problem, (4) is substituted into (2) and (3). Furthermore, the technological condition $q=\frac{y_{2}-s}{\alpha}$, can be employed to remove $q$ from the constraints and to express agent $i$ 's maximization problem as

$$
\max _{y_{1}, y_{2}, x_{i}} U\left(y_{1}, y_{2}, X\right)
$$

subject to

$$
y_{1}+\bar{p}_{s} \bar{s}+p_{q}\left(\frac{y_{2}-\bar{s}}{\alpha}\right)=I \quad \text { and } \quad-\beta\left(y_{2}-\bar{s}\right)+\alpha X=\alpha \tilde{X} .
$$

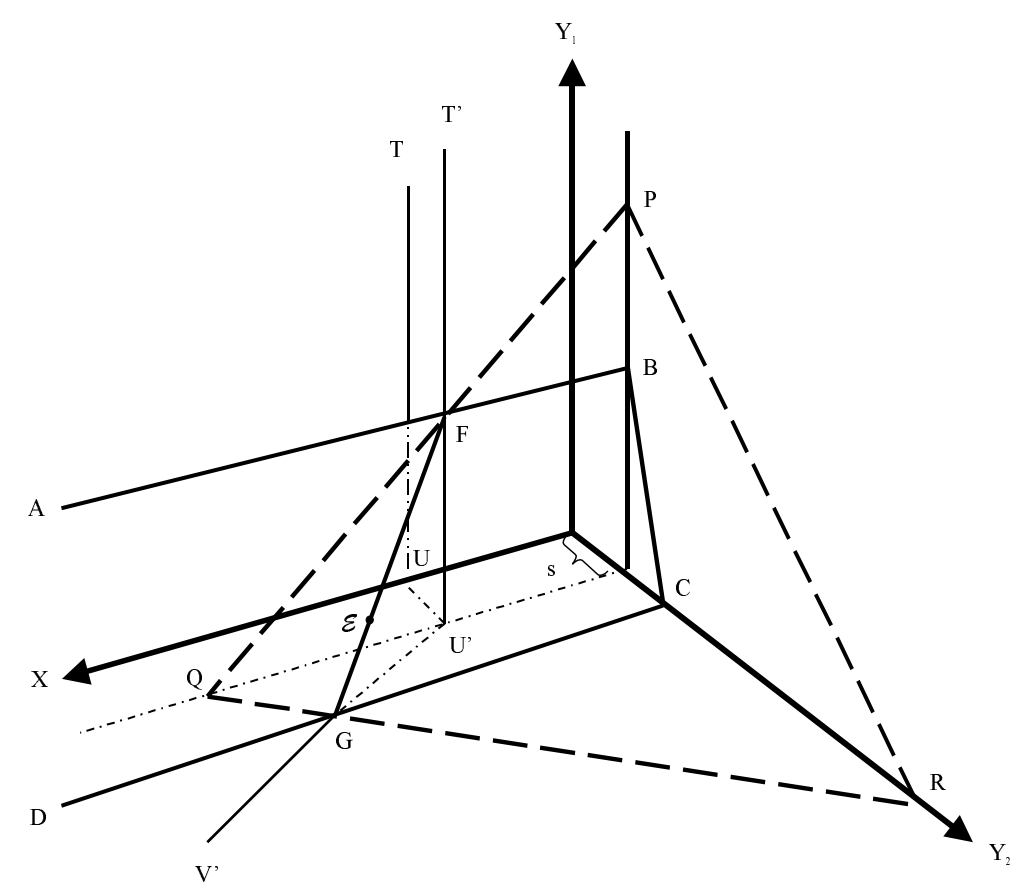

Figure 1: Constraints and Welfare Maximum.

Figure 1 illustrates the constraints restricting the agent's utility maximization: the constraint on the left-hand side is depicted by the plane ABCD and the constraint on the right-hand side is represented by plane T'U'V'. The distance between $U$ and the origin of the three axis measures the total level of the pure public characteristic generated by the other agents, $\tilde{X}$. Starting from the point $\mathrm{U}$ the agent has to buy $\bar{s}$ units of the second characteristic directly (constraint 3 ). Thus, we move to point U'; starting from 
this point the agent may buy units of the impure public good $q$ or the private good $y_{1}$. The consumption of impure public good $q$ and private good $y_{1}$ is limited by the aforementioned constraint depicted by plane ABCD. Therefore, the set of points that satisfy all three constraints lies on the line which links point $\mathrm{F}$ to the point $\mathrm{G}$. One of the indifference curves from the set of indifference curves representing the agent's convex preferences over the three characteristics is tangent to the line FG. ${ }^{9}$ The tangential point represents the allocation providing the highest attainable utility level. In Figure 1 the point $\mathcal{E}$ represents this point. Strict convexity of preferences guarantees that there is a unique point of tangency $\mathcal{E}$.

\subsection{Virtual Magnitudes}

Let us next formulate the restrictions limiting an agent's consumption by using parameters associated with familiar price-taking behaviour. Therefore, we have to introduce virtual magnitudes. These magnitudes (virtual prices and income) have to guarantee that a consumer chooses to take precisely the same amount of each commodity as he consumes on the efficient point $\mathcal{E}$. Since the externalities of the public characteristic have to be considered, not the monetary income is of main importance but the virtual income. The virtual income may also be denoted 'hypothetical income', 'full income' or 'social income'. Becker (1974: 1063) defines 'social income' as 'the sum of person's own income (his earnings, etc.) and the monetary value to him of the relevant characteristics of others". We denote the three hypothetical or virtual prices of the characteristics and the hypothetical income that would lead a price-taking agent to choose the characteristics bundle in $\mathcal{E}$ by $\psi_{1}, \psi_{2}, \psi_{3}$, and $\mu$ respectively.

If it is warranted that the virtual budget constraint containing the virtual magnitudes, $\mu=\psi_{1} y_{1}+\psi_{2} y_{2}+\psi_{3} X$, induces a price-taking agent to choose the allocation $\mathcal{E}$, the virtual magnitudes could be employed in order to form the virtual budget plane PQR that allows for abandonment of the other restricting planes in Figure 1. The points $\mathcal{E}$, $\mathrm{F}$ and $\mathrm{G}$ have to be located in the plane PQR. Thus, the virtual magnitudes depend on the location of the tangential point $\mathcal{E}$ between FG and the indifference surfaces, and the point $\mathcal{E}$ itself depends on the observed parameters of the choice problem $p_{s}, p_{q}, \mathrm{I}, \tilde{X}, \mathrm{~s}$, $\alpha, \beta$. Consequently, it is required that:

$$
\psi_{i}=f_{i}\left(y_{1}, y_{2}, X\right)=\psi_{i}\left(p_{s}, p_{q}, I, \tilde{X}, s, \alpha, \beta\right),
$$

\footnotetext{
${ }^{9}$ The set of indifference curves are not depicted in Figure 1.
} 


$$
\mu=f_{\mu}\left(y_{1}, y_{2}, X\right)=\mu\left(p_{s}, p_{q}, I, \tilde{X}, s, \alpha, \beta\right) .
$$

The functions $\psi_{i}$ reflect the slopes in the relevant directions of the indifference surface at the allocation $\mathcal{E}$. Since only the relative magnitudes are relevant, the virtual price of the first characteristic can be set equal to unity. The virtual income function $\mu$ determines the income that puts the budget plane on the level where it has the tangency point with the indifference surfaces in $\mathcal{E}$. Consequently, the plane PQR would support consumption of a price-taking agent at allocation the agent's efficient allocation $\mathcal{E}$.

\subsection{Linkage Between Exogenous and Virtual Parameters}

The linkage of the virtual magnitudes to the exogenous parameters has to meet the following requirements:

- The unit price the agent is willing to pay for the impure public good is equal to the price $p_{q}$ that is actually paid. This price has to coincide with the valuation of the characteristics generated by the unit of the impure public good. The virtual prices reflect these valuations. Per unit of the impure public good $\alpha$ units of $y_{2}$ and $\beta$ units of $X$ are provided such that:

$$
p_{q}=\alpha \psi_{2}(\Theta)+\beta \psi_{3}(\Theta) .
$$

Here, $\beta \psi_{3}(\Theta)$ represents the benefits derived from the public and $\alpha \psi_{2}(\Theta)$ the benefits derived from the private characteristics of a unit of the impure public good. Furthermore, the vector $\Theta$ gathers the observed parameters $\bar{p}_{s}, p_{q}, I, \tilde{X}, \bar{s}, \alpha, \beta$.

- In addition to the provision of $y_{2}$ by $s$, the provision of the impure public good generates $y_{2}$. The amount by which characteristic 2 is augmented by the agent's provision of the impure public good has to be proportional to the amount by which the agent augments the other agents' provision of the public characteristic:

$$
\beta\left\{D_{2}\left(\psi_{2}(\Theta), \psi_{3}(\Theta), \mu(\Theta)\right)-s\right\}=\alpha\left\{D_{3}\left(\psi_{2}(\Theta), \psi_{3}(\Theta), \mu(\Theta)\right)-\tilde{X}\right\} .
$$

$D_{k}$, with $k=2,3$, stands for the uncompensated demand functions with respect to characteristics 2 and 3 respectively.

- The virtual income of agent $i$ must be consistent with the value of the chosen allocation at $\mathcal{E}$ :

$$
\begin{aligned}
\mu(\cdot) & =y_{1}+\psi_{2}(\cdot) y_{2}+\psi_{3}(\cdot) X \\
& =y_{1}+\psi_{2}(\cdot) y_{2}+\psi_{3}(\cdot)(x+\tilde{X})
\end{aligned}
$$


Using equations (6), (7), (8) as well as the facts that $I=y_{1}+p_{q} \frac{y_{2}-s}{\alpha}+p_{s} s$ and $y_{2}=\alpha q+s$, virtual income can be expressed:

$$
\mu(\cdot)=y_{1}+p_{s} s+p_{q} q+\psi_{3} \tilde{X}+\left(\psi_{2}-p_{s}\right) s=I+\psi_{3} \tilde{X}+\left(\psi_{2}-p_{s}\right) s .
$$

The expression for $\mu(\cdot)$ in (9) deviates from the commonly employed expression of virtual income by the term $\left(\psi_{2}-p_{s}\right) s$. This is due to the fact that in the considered case the production of $s$ is allowed to be suboptimal low and the virtual price $\psi_{2}$ may not be equal to the price $p_{s}$. If no inefficient low level of $s$ prevails, the prices $p_{s}$ and $\psi_{2}$ coincide and the term $\left(\psi_{2}-p_{s}\right) s$ vanishes.

\section{Comparative Statics}

An agent can modify the extent of impure-public-good independent generation of the second private characteristic. The raised efforts in such independent generation affect the attractiveness of impure-public-good provision. The same holds with respect to price variations of the impure-public-good independent generation of the second private characteristic. The impacts of these effort and price variations are analyzed in the subsequent sections.

\subsection{Variation of the Independent Generation of the Second Char- acteristic}

In order to determine the impact of an increased impure-public-good independent generation of the second characteristic on the impure-public-good provision level, we have - in a first step - to differentiate equations (6), (7) and (9) with respect to $\mathrm{s}$. This differentiation yields

$$
\begin{aligned}
0 & =\alpha \psi_{2 s}+\beta \psi_{3 s}, \\
\beta\left(D_{22} \psi_{2 s}+D_{23} \psi_{3 s}+D_{2 \mu} \mu_{s}\right)-\beta & =\alpha\left(D_{32} \psi_{2 s}+D_{33} \psi_{3 s}+D_{3 \mu} \mu_{s}\right), \\
\mu_{s} & =\tilde{X} \psi_{3 s}+s \psi_{2 s}+\psi_{2}-p_{s} .
\end{aligned}
$$

where $D_{k 2}=\frac{\partial D_{k}}{\partial \psi_{2}}, D_{k 3}=\frac{\partial D_{k}}{\partial \psi_{3}}, D_{k \mu}=\frac{\partial D_{k}}{\partial \mu}, \psi_{2 s}=\frac{\partial \psi_{2}}{\partial s}, \psi_{3 s}=\frac{\partial \psi_{3}}{\partial s}$ and $\mu_{s}=\frac{\partial \mu}{\partial s}$. 
This system can be written:

$$
\left[\begin{array}{ccc}
\alpha & \beta & 0 \\
\beta D_{22}-\alpha D_{32} & \beta D_{23}-\alpha D_{33} & \beta D_{2 \mu}-\alpha D_{3 \mu} \\
-s & -\tilde{X} & 1
\end{array}\right]\left[\begin{array}{c}
\psi_{2 s} \\
\psi_{3 s} \\
\mu_{s}
\end{array}\right]=\left[\begin{array}{c}
0 \\
\beta \\
\psi_{2}-p_{s}
\end{array}\right]
$$

Second, we employ Cramer's rule and set $\alpha=\beta=1$ for simplicity reasons. This yields the following price and income responses:

$$
\begin{gathered}
\psi_{2 s}=\frac{\left(D_{2 \mu}-D_{3 \mu}\right)\left(\psi_{2}-p_{s}\right)-1}{\Omega}, \\
\psi_{3 s}=\frac{1-\left(D_{2 \mu}-D_{3 \mu}\right)\left(\psi_{2}-p_{s}\right)}{\Omega}, \\
\mu_{s}=\tilde{X} \frac{1-\left(D_{2 \mu}-D_{3 \mu}\right)\left(\psi_{2}-p_{s}\right)}{\Omega}+s \frac{\left(D_{2 \mu}-D_{3 \mu}\right)\left(\psi_{2}-p_{s}\right)-1}{\Omega}+\psi_{2}-p_{s},
\end{gathered}
$$

where $\Omega=\frac{\partial C_{2}}{\partial \psi_{3}}-\frac{\partial C_{2}}{\partial \psi_{2}}-\frac{\partial C_{3}}{\partial \psi_{3}}+\frac{\partial C_{3}}{\partial \psi_{2}}>0$. Here, $C$ stands for compensated demand functions.

As already mentioned above, virtual prices and income can be expressed as functions of the exogenous parameters $p_{s}, p_{q}, s, \tilde{X}, I, \alpha, \beta$. By definition virtual magnitudes have to ensure that the succeeding condition holds with respect to the uncompensated demand of the agent:

$$
D_{k}^{*}(\Theta)=D_{k}\left(\psi_{2}(\Theta), \psi_{3}(\Theta), \mu(\Theta)\right), \quad k=1,2,3 .
$$

Now, in a third step, the differentiation of (10) with respect to $s$, substitution of the calculated price and income responses and application of the Slutzky decomposition yields the following responses (for an extensive discussion of the calculations see Rübbelke (2002, 2003)):

$$
\begin{gathered}
D_{2 s}=\frac{C_{23}-C_{22}}{\Omega}+D_{2 I}\left(\psi_{2}-p_{s}\right), \\
D_{3 s}=D_{2 s}-1=\frac{C_{33}-C_{32}}{\Omega}+D_{3 I}\left(\psi_{2}-p_{s}\right) .
\end{gathered}
$$

Provided that the characteristics 2 and 3 do not represent very strong complements and as long as the virtual price $\psi_{2}$ of characteristic 2 exceeds the price $p_{s}$ for direct provision of this characteristic, the response of the demand $D_{2}$ to an increase in $s$ is positive. This is on the one hand due to the fact that - provided both characteristics behave like normal goods 
- an income rise will increase the demand for both characteristics and hence, the second terms on the right-hand sides of both responses are positive (see Rübbelke $(2002,2003)$ ). On the other hand this is due to the exclusion of the case of very strong complements, and therefore $C_{23}>C_{22} \cdot{ }^{10}$ The latter causes the first term of (11) also to be positive.

The sign of the response of $D_{3}$ is ambiguous since the difference between $C_{33}$ and $C_{23}$ is negative. Hence, it is ambiguous whether the provision of the impure public good will rise or decrease.

Yet, if it is assumed that $p_{s}$ equals $\psi_{2}$, then the response $D_{2 s}$ is definitely positive and the response in $D_{3 s}$ is definitely negative. Then, the overall response of the provision of the impure public good is negative since direct provision of the second characteristic crowds out indirect provision via impure public good provision.

\subsection{Variation of the Price of the Technology Which Generates the Second Characteristic Independently}

Let us next analyze the impact of a change in the price of the impure-public-good independent generation of the second private characteristic. By differentiation of equations (6), (7) and (9) with respect to $p_{s}$, we get

$$
\begin{aligned}
0 & =\alpha \psi_{2 p_{s}}+\beta \psi_{3 p_{s}}, \\
\beta\left(D_{22} \psi_{2 p_{s}}+D_{23} \psi_{3 p_{s}}+D_{2 \mu} \mu_{p_{s}}\right) & =\alpha\left(D_{32} \psi_{2 p_{s}}+D_{33} \psi_{3 p_{s}}+D_{3 \mu} \mu_{p_{s}}\right), \\
\mu_{p_{s}} & =\psi_{3 p_{s}} \tilde{X}+\left(\psi_{2 p_{s}}-1\right) s .
\end{aligned}
$$

This can be expressed:

$$
\left[\begin{array}{ccc}
\alpha & \beta & 0 \\
\beta D_{22}-\alpha D_{32} & \beta D_{23}-\alpha D_{33} & \beta D_{2 \mu}-\alpha D_{3 \mu} \\
-s & -\tilde{X} & 1
\end{array}\right]\left[\begin{array}{c}
\psi_{2 p_{s}} \\
\psi_{3 p_{s}} \\
\mu_{p_{s}}
\end{array}\right]=\left[\begin{array}{c}
0 \\
0 \\
-s
\end{array}\right]
$$

By application of Cramer's rule and setting $\alpha=\beta=1$, we get the following price and income responses:

$$
\psi_{2 p_{s}}=\frac{-s\left(D_{2 \mu}-D_{3 \mu}\right)}{\Omega}
$$

\footnotetext{
${ }^{10}$ Consider, that compensated cross-price responses are symmetric; see Deaton and Muellbauer (1980: 43-45) as well as Cornes (1992: 71-72).
} 


$$
\begin{gathered}
\psi_{3 p_{s}}=\frac{s\left(D_{2 \mu}-D_{3 \mu}\right)}{\Omega} \\
\mu_{p_{s}}=\tilde{X} \frac{s\left(D_{2 \mu}-D_{3 \mu}\right)}{\Omega}+s \frac{-s\left(D_{2 \mu}-D_{3 \mu}\right)}{\Omega}-s .
\end{gathered}
$$

By differentiating (10) with respect to $p_{s}$, applying the Slutzky decomposition and substituting the calculated price and income responses, we get:

$$
D_{2 p_{s}}=D_{3 p_{s}}=s \frac{\left(C_{22}-C_{23}\right) D_{3 \mu}+\left(C_{33}-C_{32}\right) D_{2 \mu}}{\Omega}=-\left(s D_{2 I}\right) .
$$

Thus, a rise in the price for direct acquisition of characteristic 2 induces only an income effect. This is in line with Neary and Roberts (1980: 34) who point out that an increase in the price of a rationed good simply forces the consuming household to pay more for the rationed consumption level. Because the uncompensated demand for characteristics 2 and 3 decrease, the agent will lower his impure-public-good provision level. However, the latter result is a consequence of the model assumption that the independent generation of joint products cannot be substituted by a generation which is associated with the impure public good production. Otherwise, the higher opportunity cost of the independent generation may cause the impure-public-good provision level to rise.

\section{Numerical Simulation}

Since our analytical solutions are not clear-cut, we employ the impure-public-good example of climate policy in Germany to numerically simulate the effects of alternative technologies in a parameterized model. The simulation model reveals directions of impacts where the analytical model has ambiguous results and gives insights into the magnitude of effects associated with changes in the quantity and the price of the impure-public-good independent generation of the second private characteristic.

\subsection{Impure Public Goods: The Example of Climate Policy}

Since climate policy, e.g., in the shape of a $\mathrm{CO}_{2}$ tax, provides pure public as well as private characteristics, it can be regarded as an impure public good. Pure public and private characteristics are provided by climate policy (see Figure 2).

The pure public characteristic subsumes the effects generated by climate policy that can be enjoyed globally, irrespective of which country abates. The pure public characteristic provided by climate policy is climate protection. No country can be excluded from 
its consumption and it exhibits non-rivalry. Benefits resulting from climate protection are the primary benefits of climate policy.

Climate policy also provides some private characteristic with purely local/regional influence that can be exclusively enjoyed by countries/regions generating climate policy. The benefits, which countries enjoy from consuming the private characteristic are the ancillary or secondary benefits.

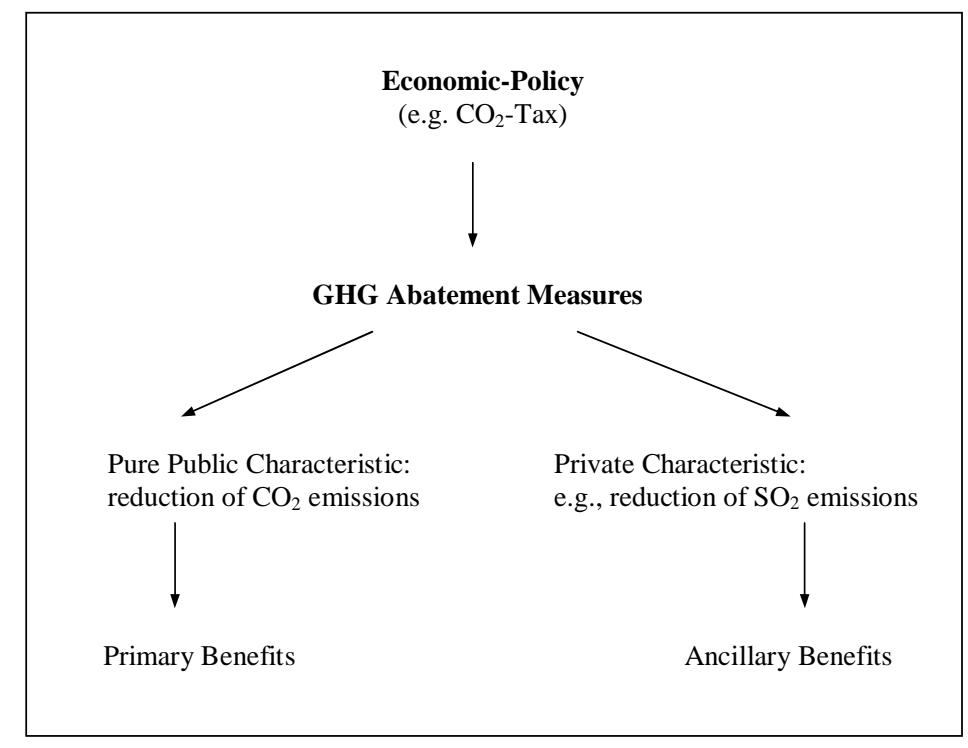

Figure 2: Primary and Ancillary/Secondary Benefits.

Yet, we have to take account of the fact that the ancillary benefits can also be generated independently of climate policy. Desulphurization installations, for example, reduce the emission of $\mathrm{SO}_{2}$ independently of climate policy. So, the higher the level of such installations, the lower will be the (marginal) ancillary benefits of climate policy. Subsequently, we will focus on the ancillary effect of $\mathrm{SO}_{2}$ emission mitigation.

\subsection{The Simulation Model}

This section describes the data used to parameterize the analytical model presented in Section 2 in order to simulate numerically the effects of changes in the quantity and prices of the alternative technology which are analyzed analytically in Section 3. For the calibration we consider the example of climate policy in Germany in the year 2010 where $\mathrm{CO}_{2}$ emission reductions is the impure public good and $\mathrm{SO}_{2}$ emission reduction is the 
private environmental good.

To parameterize the benchmark demand for the impure public good and the private environmental good we assume that international obligations are revealed preferences for the national provision of the two goods, $\mathrm{CO}_{2}$ and $\mathrm{SO}_{2}$ emission reductions. The Kyoto Protocol to the United Nations Framework Convention on Climate Change (UNFCCC) which entered into force in February 2005 imposes greenhouse gas emission limits for industrialized countries (UNFCCC (1997)). In May 2002, the European Union (EU) ratified the Kyoto Protocol committing itself to a reduction of EU-wide greenhouse gas emissions by $8 \%$ vis-à-vis 1990 emission levels during 2008-2012. The aggregate EU reduction requirement has been redistributed among individual Member States according to an EU-internal Burden Sharing Agreement resulting in a reduction requirement for Germany of 21\% (CEC (1999a)). Given $\mathrm{CO}_{2}$ emissions of $952 \mathrm{MtCO}_{2}$ in Germany and 3,068 $\mathrm{MtCO}_{2}$ in the $\mathrm{EU}$ in 1990, the emission targets associated with the Kyoto Protocol amount to $752 \mathrm{MtCO}_{2}$ for Germany and 2,823 $\mathrm{MtCO}_{2}$ for the EU. Projected emissions for 2010 (the base-year for our model simulations) amount to $839 \mathrm{MtCO}_{2}$ for Germany and 3,311 $\mathrm{MtCO}_{2}$ for the EU (CEC (2001a)). This yields a benchmark demand for $\mathrm{CO}_{2}$ emission reduction for Germany of $q=87 \mathrm{MtCO}_{2}$. We assume that real emission reductions are only achieved in Europe and that the Kyoto Protocol is in addition reduced to symbolic policy (Löschel and Zhang (2003)). The other agents' provision of the impure public good is then $\tilde{Q}=401 \mathrm{MtCO}_{2}$. In 1990, SO $\mathrm{O}_{2}$ emissions were $5280 \mathrm{ktSO}$. They are projected to decrease to $740 \mathrm{ktSO}_{2}$ until 2010 (CEC (2001a)). Given the German $\mathrm{SO}_{2}$ emission target of $520 \mathrm{ktSO}_{2}$ in 2010 stated in the EU directive on National Emission Ceilings for certain atmospheric pollutants (CEC (2001b)), the demand for the private environmental good $\mathrm{SO}_{2}$ emission reduction is $y_{2}=220 \mathrm{ktSO} \mathrm{S}_{2}$.

The parameter $\alpha$ that describes the extent of $\mathrm{SO}_{2}$ emission reduction that is generated through the reduction of $\mathrm{CO}_{2}$ emissions is estimated through simulation experiments with the GEM-E3 model for Europe (Capros et al. (1997)). It is a full scale general equilibrium model for 15 European countries, linked through endogenous bilateral trade. The environmental module of GEM-E3 concentrates on three environmental problems: (i) global warming, (ii) problems related to the deposition of acidification emissions and (iii) ambient air quality linked to acidifying emissions and tropospheric ozone concentration. Hence, it considers energy-related emissions of primary pollutants $\mathrm{CO}_{2}, \mathrm{NO}_{\mathrm{X}}, \mathrm{SO}_{2}$, VOC and particulates. There are two mechanisms of emission reduction explicitly specified in the model: substitution between fuels and between energetic and non-energetic inputs 
and emission reduction due to a decline in production and consumption. We simulate with the GEM-E3 model a scenario where Germany reaches its burden sharing emission level of $\mathrm{CO}_{2}$ through a domestic permit system. In this scenario the marginal cost of $\mathrm{CO}_{2}$ abatement (price of the impure public good) is $p_{q}=29.0$ Eurog7 $_{97} / \mathrm{tCO}_{2}$ and $\alpha=$ $1.30 \mathrm{ktSO} / \mathrm{MtCO}_{2}$. The amount of $\mathrm{SO}_{2}$ emission reduction that is generated through reduction of $\mathrm{CO}_{2}$ to reach the German Kyoto obligations is $113 \mathrm{ktSO}$. The benchmark quantity of $\mathrm{SO}_{2}$ emission reduction that has to be provided independent of climate policy to reach the EU National Emission Ceilings is therefore $s=107 k t \mathrm{SO}_{2}$.

The marginal costs of $\mathrm{SO}_{2}$ reductions by different control technologies for Germany in the current legislation (baseline) scenario for 2010 is taken from the Regional Air Pollution Information and Simulation (RAINS) model (Cofala and Syri (1998)), which is applied as a scenario analysis tool in the context of the international negotiations under the UN/ECE Convention on Long-range Transboundary Air Pollution and for the development of the acidification and ozone strategies of the European Union (Alcamo et al. (1990)). RAINS concentrates on the technical emission control options, which do not imply structural changes of the energy system. Five broad groups of technical emission control options are distinguished: (i) the use of low-sulphur fuels, including fuel desulphurization, (ii) in-furnace control of $\mathrm{SO}_{2}$ emissions, (iii) conventional wet flue gas desulphurization processes, (iv) advanced, high efficiency methods for capturing sulphur from the flue gas, and (v) measures to control process emissions. The marginal cost for $107 k t \mathrm{SO}_{2}$ reduction or equivalently the benchmark price of the alternative technology amounts to $p_{s}=4,920$ Euro $_{97} / t_{S O_{2}}$.

The total budget is taken from the macroeconomic assumption for Germany in the baseline scenario "European Environmental Priorities" (Capros et al. (2000), CEC (1999b)): $I=2,561,000$ million Euro 97 .

We employ a linear logarithmic utility function of the form

$$
U=\gamma_{1} \ln y_{1}+\gamma_{2} \ln y_{2}+\gamma_{3} \ln X
$$

and calibrate the parameter in the utility function such that our benchmark data set is replicated. Table 1 summarizes the benchmark parameter values for the numerical simulations. In the simulation runs we then vary the benchmark values of the quantity $s$ and the price $p_{s}$ of the alternative technology. 


\begin{tabular}{|cl|r|rl|r|}
\hline$y_{1}$ & (in M Euro 97$)$ & $2,557.95$ & $X$ & $\left(\mathrm{MtCO}_{2}\right)$ & 488 \\
\hline$p_{y}$ & $\left(\right.$ Euro $\left._{97}\right)$ & 1 & $I$ & $\left(\right.$ in 000 $\left.\mathrm{M} \mathrm{EurO}_{97}\right)$ & 2,561 \\
\hline$s$ & $\left(\mathrm{ktSO}_{2}\right)$ & 107 & $\alpha \quad\left(\mathrm{ktSO}_{2} / \mathrm{MtCO}_{2}\right)$ & 1.30 \\
\hline$p_{\mathrm{s}}$ & $\left(\right.$ Euro $\left._{97} / \mathrm{CO}_{2}\right)$ & 4,920 & $\beta$ & 1 \\
\hline$q$ & $\left(\mathrm{MtCO}_{2}\right)$ & 87 & $U$ & 29.52 \\
\hline$p_{\mathrm{q}}$ & $\left(\mathrm{EurO}_{97} / \mathrm{CO}_{2}\right)$ & 29 & $\Psi_{1}$ & $1,6730.77$ \\
\hline$\tilde{\mathrm{Q}}$ & $\left(\mathrm{MtCO}_{2}\right)$ & 401 & $\Psi_{2}$ & 7.25 \\
\hline$\tilde{X}$ & $\left(\mathrm{MtCO}_{2}\right)$ & 401 & $\Psi_{3}$ & $2,565.17$ \\
\hline$x$ & $\left(\mathrm{MtCO}_{2}\right)$ & 87 & $\mu$ & \\
\hline
\end{tabular}

Table 1: Benchmark Parameter Values.

\section{Simulation Results and the Impure Public Good Model}

The simulation yielded some interesting results which either confirm our analytical investigation or reveal the direction of impacts given ambiguous results in the analytical model. Furthermore, our findings turn out to have surprising implications. In contrast to the analytical model, the simulation study allows us to have an explicit look on changes in utility levels induced by changes of individual parameters.

The effects induced by a change in the price of the second private good are in line with the predictions of the impure public good model (see Figure 3). A rising price causes the consumption of all goods $y_{1}, y_{2}, q$ (as well as welfare) to decline, which is due to the negative income effect exerted by the increase in the price of the rationed good. The income effect is the only effect resulting from the increase in $p_{s}$, as Neary and Roberts (1980) as well as our analytical analysis show. Therefore, the simulation result is not very surprising but confirms the appropriateness of our analytical approach. We can observe from Figure 3, how virtual income and utility level decline with rising price levels of the rationed good.

In contrast to the unambiguous impacts of the increase in $p_{s}$ predicted by the analytical impure public good model, the model's forecasts of effects of a change in the level $s$ of the consumption of the second private good on the impure public good provision were ambiguous. The analytical model predicted that the increase in $s$ may raise or reduce the provision level $q$ of the impure public good. In our simulation model, we find a negative 


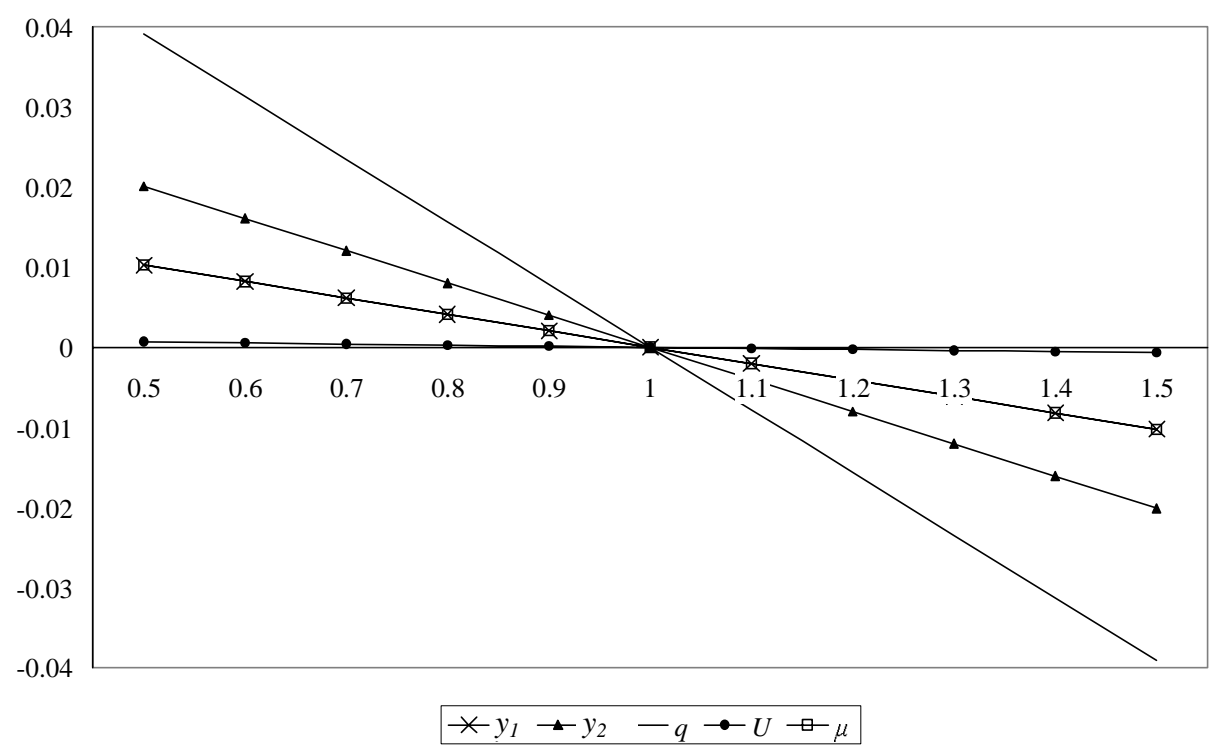

Figure 3: Effects induced by a change in the price of the second private good $p_{s}$ (percentage change vs. benchmark value).

effect of the increase in the second private good consumption level on impure public good provision $q$. This decline in impure public good provision is accompanied by a rise in welfare and virtual income levels. Yet, utility levels increase at a declining pace with rising levels of $s$.

A very interesting and surprising result is that variations in the price $p_{s}$ of the rationed second private good do only have minor negative impacts on climate protection levels $q$ compared to variations in the levels of the rationed good $s$ (see Figures 3 and 4 ). With respect to our example of climate policy, this means that an increase in the prices of technologies independently (of climate policy) generating private ancillary effects of climate policy (like desuphurisation installations reducing $\mathrm{SO}_{2}$ emissions) does not have a very strong negative impact on climate protection levels. In contrast, regulations prescribing higher standards for pollution control of local or regional air pollutants tend to have a significant negative impact on climate protection levels. Nevertheless, these regulations tend to improve welfare, although the welfare improvement rises at a slower pace with increasing emission control. The welfare improvement is due to the suboptimal low provision of $\mathrm{SO}_{2}$ emission control in the baseline scenario: with increasing control levels, the marginal benefits of $\mathrm{SO}_{2}$ emission control decline. 


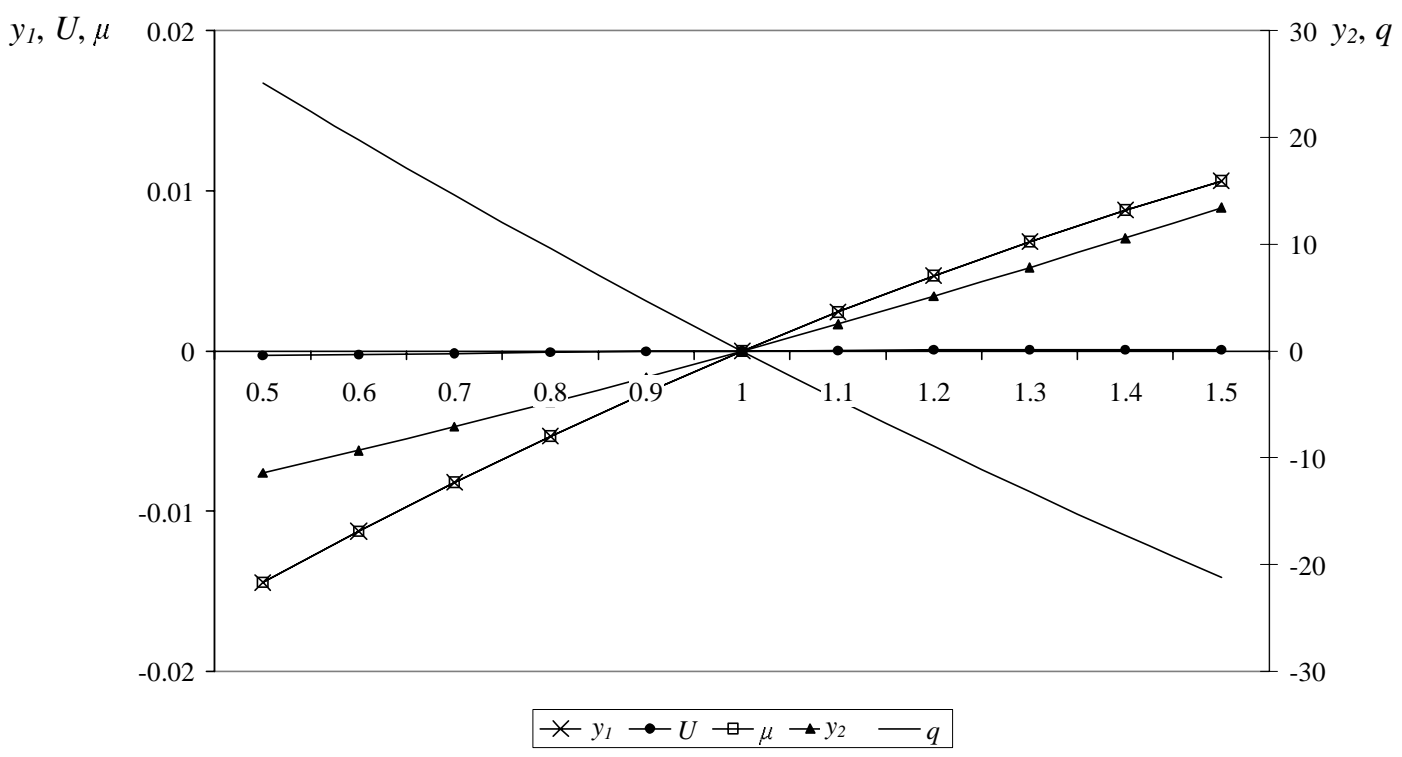

Figure 4: Effects induced by a change in the quantity $s$ of the second private good (percentage change vs. benchmark value).

\section{Conclusions}

There exists a great variety of application options for impure public good models. In fact, almost every public goods has an impure public character especially when we regard international public goods.

The analytical model we presented in Section 2 addresses such impure public goods. Yet, in many cases, the private characteristic of the impure public good can be produced by different means. Therefore, in contrast to standard public good models, we introduced a third technology, generating the private characteristic of the impure public good independently. We supposed this technology to be rationed and it was in the center of the focus of our analysis. We derived by means of our analytical model the impact this technology's application and price have on the provision level of the impure public good. Therefore, we investigated influences which are omitted in standard pure public good models, which do not take account of private characteristics associated with public good provision.

In order to check and refine the results of the analytical study, we employed a simulation model for the example of climate policy in Germany. Here, climate protection represents the global public characteristic of the impure public good 'climate policy', 
while $\mathrm{SO}_{2}$ abatement represents the private characteristic, from an individual country's point of view. In fact, most options to reduce the emission levels of the greenhouse gas $\mathrm{CO}_{2}$ turn out to be simultaneously reducing the emissions of $\mathrm{SO}_{2}$. However, the emissions of $\mathrm{SO}_{2}$ can also be mitigated independently of climate policy by means of desulphurisation installations, which represent the technology independently generating the private characteristic of the impure public good.

We had to take account of the fact that the degrees of publicness vary with divergent entities considered. So, in standard analyses local/regional air pollution reductions represent a public good for inhabitants of the respective local/regional area. Yet, on the international scale we considered, abatement of local/regional air pollution turns out to become a private good from an individual country's point of view (while still representing a public good for individual inhabitants living in the area enjoying higher air quality).

The results of the simulations confirmed the findings of the analytical model. So, the increase of the price of climate-policy independent $\mathrm{SO}_{2}$ abatement exerts a negative income effect. Consequently, the abatement has a negative impact on impure public good provision, and therefore on climate protection levels. As our explicit illustration of welfare effects in the framework of the simulation model shows, welfare levels unambiguously decline with the considered price increases.

In contrast to the ambiguous results of our analytical representation with respect to the impacts of an increase in the provision of the rationed good, we found unambiguous effects of such an increase in our simulation model. The provision of the impure public good decrease with increasing levels of the rationed private good. Due to the inefficiently low provision level in the baseline scenario, the increased production of the rationed good caused an improvement of welfare.

Interestingly, the impact of the price increase of $\mathrm{SO}_{2}$ abatement has minor effects on climate protection levels compared to increases in the climate-policy-independent enhancement of $\mathrm{SO}_{2}$ abatement. Consequently, it turns out to be likely that stricter regulations on air-pollution may cause the attractiveness of climate policy to decline significantly, while price increases of this control tend to have only minor effects. 


\section{$7 \quad$ References}

Alcamo J., Shaw R. and Hordijk L. (1990), The RAINS Model of Acidification: Science and Strategies in Europe, Kluwer (Dordrecht).

Andreoni, J. (1986), Essays on Private Giving to Public Goods, UMI (Michigan).

Andreoni, J. (1989), "Giving with Impure Altruism: Applications to Charity and Ricardian Equivalence," Journal of Political Economy, 97, 1447-1458.

Andreoni, J. (1990), "Impure Altruism and Donations to Public Goods: A Theory of Warm-Glow Giving," The Economic Journal, 100, 464-477.

Baumol, W.J. and Bowen, W.G. (1966), Performing Arts: The Economic Dilemma, Twentieth Century Fund (New York).

Becker, G.S. (1974), "A Theory of Social Interactions," Journal of Political Economy, 82, 1063-1093.

Capros, P., Georgakopoulos, T., Van Regemorter, D., Proost, S., Schmidt, T. and Conrad, K. (1997), "The GEM-E3 Model for the European Union," Journal of Economic E Financial Modelling, 4, 51-160.

Capros, P., Mantzos, L., Pearce, D.W., Howarth, A., Sedeem, C., and Strengers, B.J. (2000), Technical Report on Climate Change, European Environmental Priorities: an Integrated Economic and Environmental Assessment, RIVM Report, May 2000.

CEC (Commission of the European Communities) (1999a), Preparing for Implementation of the Kyoto Protocol, Commission Communication to the Council and the Parliament, COM (99)230, 19 May 1999 (Brussels).

CEC (Commission of the European Communities) (1999b), European Union Energy Outlook to 2020, Office for Official Publications of the European Communities (Luxembourg).

CEC (Commission of the European Communities) (2001a), Environment 2010: Our Future, Our Choice, The Sixth Environment Action Programme of the European Community, $\mathrm{COM}(2001) 31$ (Brussels).

CEC (Commission of the European Communities) (2001b), "Directive 2001/81/EC of the European Parliament and of the Council of 23 October 2001 on National Emission Ceilings for Certain Atmospheric Pollutants," in: Official Journal of the European Communities, L 309, 22-30. 
Cofala, J. and Syri, S. (1998), Sulfur Emissions, Abatement Technologies and Related Costs for Europe in the RAINS Model Database, International Institute for Applied Systems Analysis Interim Report, IR-98-035 (Laxenburg).

Cornes, R.C. (1992), Duality and Modern Economics, Cambridge University Press (Cambridge).

Cornes, R.C. and Sandler, T. (1984), "Easy Riders, Joint Production, and Public Goods," The Economic Journal, 94, 580-598.

Cornes, R.C. and Sandler, T. (1994), "The Comparative Static Properties of the Impure Public Good Model," Journal of Public Economics, 54, 403-421.

Deaton, A. and Muellbauer, J. (1980), Economics and Consumer Behaviour, Cambridge University Press (Cambridge).

Diamond, L. (2002), "Reinventing Foreign Aid in the National Interest," The Hoover Weekly Essay series, Hoover Institution, Stanford University, 1-3.

Dubin, J.A. and Navarro, P. (1988), "How Markets for Impure Public Goods Organize: The Case of Household Refuse Collection," Journal of Law, Economics, and Organization, 4, 217-242.

Ihori, T. (1992), "Impure Public Goods and Transfers in a Three-Agent Model," Journal of Public Economics, 48, 385-401.

Ihori, T. (1994), "Immiserizing Growth with Interregional Externalities of Public Goods," Regional Science and Urban Economics, 24, 485-496.

Kaul, I., Grunberg, I. and Stern, M. (1999), Global Public Goods, Oxford University Press (New York).

Kingma, B.R. and McClelland, R. (1995), "Public Radio Stations Are Really, Really Not Public Goods: Charitable Contributions and Impure Altruism," Annals of Public and Cooperative Economics, 66, 65-76.

Kotchen, M.J. (2005), "Impure Public Goods and the Comparative Statics of Environmentally Friendly Consumption," Journal of Environmental Economics and Management, 49, 281-300.

Löschel, A. and Zhang, Z. (2002), "The Economic and Environmental Implications of the US Repudiation of the Kyoto Protocol and the Subsequent Deals in Bonn and Marrakech," Review of World Economics - Weltwirtschaftliches Archiv, 138, 711-746. 
Neary, J.P. and Roberts, K.W.S. (1980), "The Theory of Household Behaviour Under Rationing," European Economic Review, 13, 25-42.

Pearce, D. (2000), "Policy Frameworks for the Ancillary Benefits of Climate Change Policies," in: Ancillary Benefits and Costs of Greenhouse Gas Mitigation, OECD (Paris), 517-560.

Posnett, J. and Sandler, T. (1986), "Joint Supply and the Finance of Charitable Activity," Public Finance Quarterly, 14, 209-222.

Rübbelke, D.T.G. (2002), International Climate Policy to Combat Global Warming An Analysis of the Ancillary Benefits of Reducing Carbon Emissions, Edward Elgar (Cheltenham).

Rübbelke, D.T.G. (2003), "An Analysis of Differing Abatement Incentives," Resource and Energy Economics, 25, 269-295.

Rübbelke, D.T.G. (2005), "Differing Motivations for Terrorism," Defence and Peace Economics, 16, 19-27.

Sandler, T. (1996), "A Game-Theoretic Analysis of Carbon Emissions," in The Political Economy of Environmental Protection - Analysis and Evidence, edited by Congleton, R.D., Ann Arbor - The University of Michigan Press, 251-272.

Sandler, T. (1997), Global Challenges - An Approach to Environmental, Political, and Economic Problems, Cambridge University Press (Cambridge).

Sandler, T. and Murdoch, J.C. (1990), "Nash-Cournot or Lindahl Behavior? An Empirical Test for the NATO Allies," Quarterly Journal of Economics, 105, 875-894.

Sandler, T. and Hartley, K. (2001), "Economics of Alliances: The Lessons for Collective Action," Journal of Economic Literature, XXXIX, 869-896.

UNFCCC (United Nations Framework Convention on Climate Change) (1997), Kyoto Protocol to the United Nations Framework Convention on Climate Change, FCCC/CP /L.7/Add1, Kyoto. 